\begin{tabular}{|c|c|}
\hline Title & $\begin{array}{l}\text { A New A daptive Mesh Refinement Method in FEA Based on Magnetic Field Conservation at Elements Interfaces and } \\
\text { Non-Conforming Mesh Refinement Technique }\end{array}$ \\
\hline Author(s) & Noguchi, So; Naoe, Takuto; Igarashi, Hajime; Matsutomo, Shinya; Cingoski, V Iatko; A hagon, A kira; Kameari, A kihisa \\
\hline Citation & $\begin{array}{l}\text { IEEE transactions on magnetics, 53(6), } 7201904 \\
\text { https://doi.org/10.1109/T MA G.2017.2655049 }\end{array}$ \\
\hline Issue Date & 2017-06 \\
\hline Doc URL & http:/hdl.handle.net/2115/66970 \\
\hline Rights & $\begin{array}{l}\text { C } 2017 \text { IEEE. Personal use of this material is permitted. Permission from IEEE must be obtained for all other uses, in } \\
\text { any current or future media, including reprinting/republishing this material for advertising or promotional purposes, } \\
\text { creating new collective works, for resale or redistribution to servers or lists, or reuse of any copyrighted component of } \\
\text { this work in other works. }\end{array}$ \\
\hline Type & article (author version) \\
\hline File Information & ErrorEstimation_merged.pdf \\
\hline
\end{tabular}

Instructions for use 


\title{
A New Adaptive Mesh Refinement Method in FEA Based on Magnetic Field Conservation at Elements Interfaces and Non- conforming Mesh Refinement Technique
}

\author{
So Noguchi ${ }^{1,2,3,4}$, Takuto Naoe $^{1}$, Hajime Igarashi ${ }^{1}$, Shinya Matsutomo ${ }^{5}$, Vlatko Cingoski ${ }^{6}$, Akira Ahagon ${ }^{7}$, and \\ Akihisa Kameari ${ }^{7}$ \\ ${ }^{1}$ Graduate School of Information Science and Technology, Hokkaido University, Sapporo 060-0815, Japan \\ ${ }^{2}$ Plasma Science and Fusion Center, Massachusetts Institute of Technology, Cambridge, MA 02139, USA \\ ${ }^{3}$ National High Magnetic Field Laboratory, Tallahassee, FL 32310, USA \\ ${ }^{4}$ Florida State University, Tallahassee 32310, USA \\ ${ }^{5}$ National Institute of Technology, Niihama College, Niihama 792-8580, Japan \\ ${ }^{6}$ Faculty of Electrical Engineering, University “Goce Delcev” - Stip, Skopje 1000, Macedonia \\ ${ }^{7}$ Science Solutions International Laboratory, Inc., Tokyo 153-0065, Japan
}

\begin{abstract}
Mesh quality strongly affects the solution accuracy in electromagnetic finite element analysis. Hence, the realization of adequate mesh generation becomes a very important task. Several adaptive meshing methods for automatic adjustments of the mesh density in accordance with the shape and complexity of the analyzed problem, have been proposed. However, the most of them are not enough robust, some are quite laborious and could not be universally used for adaptive meshing of complex analysis models.

In this paper, a new adaptive mesh refinement method based on magnetic field conservation at the border between finite elements is proposed. The proposed error estimation method provides easy mesh refinements, generates smaller element within regions with large curvature of the magnetic flux lines. The proposed adaptive mesh refinement method based on non-conforming edge finite elements, which could avoid generation of flat- or ill-shaped elements, was applied to a simple magnetostatic permanent magnet model. To confirm the validity and accuracy, the obtained results were compared with those obtained by means of the Zienkiewich-Zhu (ZZ) error estimator. The results show that the computational error using the proposed method was reduced down to $1.0 \%$ compared with that of the $\mathrm{ZZ}$ method which yields error of $8.6 \%$, for the same model.
\end{abstract}

Index Terms - Adaptive meshing, element surface integral term, error estimation, finite element analysis.

\section{INTRODUCTION}

FINITE Element Method (FEM) is one of the most simulation methods in electromagnetics. However, an eternal problem is how to generate adequate mesh to enhance simulation accuracy, and reduce computation time and used memory. Hence, to tackle this problem, some adaptive meshing techniques have already been investigated [1], [2].

Two major techniques are required for obtaining good adapting meshing method: (1) a good error estimation method, and (2) an adequate mesh refinement technique, of which the first one has crucial importance. Although the ZienkiewiczZhu (ZZ) method [3] is widely used as an error estimator, recently some other error estimation methods based on the local (element) error estimators obtained from the local verification (residual) of the FEM analysis and the conservation of the magnetic field $\boldsymbol{H}$ at the interface between two elements are very promising [4]-[6]. These newly introduced error estimator have proven superior to the $\mathrm{ZZ}$ method, pointing out that the magnetic field conservation could be successfully used as an error estimator in FEM analysis. However, no results have already been reported on a

Manuscript received November 20, 2016. Corresponding author: S. Noguchi (e-mail: noguchi@ssi.ist.hokudai.ac.jp).

Color versions of one or more of the figures in this paper are available online at http://ieeexplore.ieee.org.

Digital Object Identifier (inserted by IEEE). development of an adaptive FEA using this superior error estimator based on the magnetic field conservation.

Mesh refinement scheme is also important in an adaptive meshing. After estimation of the errors, elements estimated to have a large error need to be refined. As a mesh refinement technique for triangular or tetrahedral meshes, the Delaunay triangulation method is conventionally used. However, using the Delaunay triangulation method, many ill-shaped elements such as flat, distorted, or inside-out elements are generated. Therefore, we proposed a new mesh refinement method using the non-conforming finite element technique [7], [8].

In this paper, the authors proposed a new adaptive meshing method based on the magnetic field conservation between two elements and the non-conforming edge finite element technique. The proposed method results with a generation of a suitably coarse mesh with less number of finite elements and less computational cost.

\section{ADAPTIVE MESHING METHOD}

\section{A. Adaptive Meshing Method}

Fig. 1 shows the flow of common adaptive meshing finite element analysis (FEA). A bad error estimator could lead to generation of an unnecessary large number of elements with low accuracy result. Nevertheless, a good error estimator produces a fine mesh with adequately large and well distributed finite elements, resulting with high accurate 
computational results.

The commonly used ZZ error estimator [3] performs error estimation based on the field energy:

$$
\lambda_{e}=\frac{\frac{1}{2} \int\left(B_{e}-\bar{B}_{e}\right) \cdot v\left(B_{e}-\bar{B}_{e}\right) \mathrm{d} e}{\frac{1}{2} \int \bar{B}_{e} \cdot v \overline{\boldsymbol{B}}_{e} \mathrm{~d} e}
$$

where $\lambda_{e}, \boldsymbol{B}_{e}, \overline{\boldsymbol{B}}_{\boldsymbol{e}}$, and $v$ are the estimated error, the magnetic field obtained from FEA, the magnetic field estimated from the neighboring elements, and the magnetic reluctivity, respectively. Although the $\mathrm{ZZ}$ method is a very good error estimator, often in the air region far from objects, this method results with gratuitously subdivided smaller elements (e.g. Fig. 7(c)), that unwontedly increase the number of elements and the computational time, while in the same time, does not improve the computational accuracy.

As seen from Fig. 1, the mesh improvement procedure is the second important task for obtaining highly accurate solutions. Commonly, the Delaunay triangulation method is used for mesh improvement. It is a well-known, stable, and powerful tool for making triangular or tetrahedral meshes. However, when it is used for mesh refinement in an adaptive FEA, many ill-quality elements are generated. As shown in Fig. 2, even if the shapes of a parent element is well, after subdivision, newly generated offspring elements become illshaped, or if a flat parent element is subdivided, flatter offspring elements are generated. Subsequently, with each subsequent subdivision, the quality of elements downgrades, making the Delaunay triangulation method unsuitable for the mesh improvement in an adaptive FEA.

Therefore, in this paper we implemented a new adaptive analysis method which utilizes a new error estimator based on

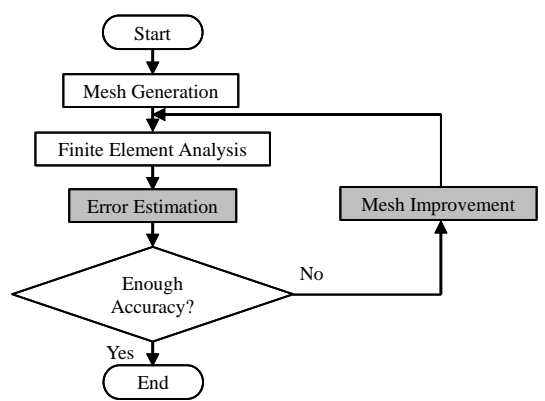

Fig. 1. Flowchart of conventional adaptive meshing FEA.

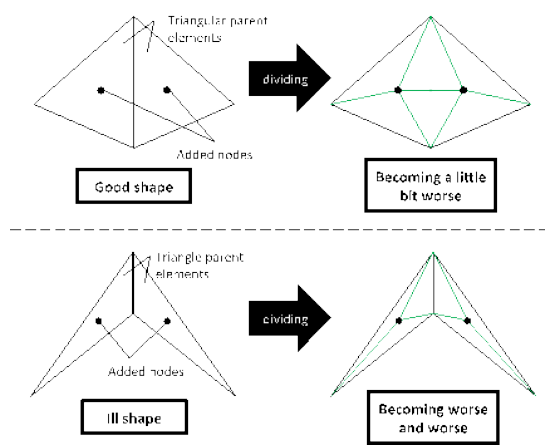

Fig. 2. Well-shaped parent element produce ill-shaped offspring elements (upper). Ill-shaped parent element produce ill-shaped offspring elements (lower). the magnetic field conservation on the interface between two elements [4], and a new mesh refinement scheme based on the non-conforming edge-based FEA [7], [8].

\section{B. Proposed Error Estimator based of the Magnetic Field Conservation}

The governing equation for magnetostatic problems is: $\nabla \times(\boldsymbol{W} \times \boldsymbol{A})=\boldsymbol{J}_{0}$

where $\boldsymbol{A}$ and $\boldsymbol{J}_{0}$ are the magnetic vector potential and the source current, respectively. Applying the weighted residual method, from (2) the following equation could be obtained:

$\int_{v} w \cdot \nabla \times(\mathcal{W} \times \boldsymbol{A}) \mathrm{d} v=\int_{v} w \cdot J_{0} \mathrm{~d} v$

where $\boldsymbol{w}$ and $v$ are the vector interpolation function and the integration volume, respectively. With integration by parts, the left term of (3) becomes:

$\int_{v} w \cdot \nabla \times(\boldsymbol{D} \times A) \mathrm{d} v$

$=\int_{v} \boldsymbol{v}(\nabla \times w) \cdot(\nabla \times A) \mathrm{d} \boldsymbol{v}+\int_{S} \boldsymbol{v} \cdot[(\nabla \times A) \times w] \mathrm{d} S$

where $S$ and $\boldsymbol{n}$ are the integration surface and the unit vector normal to the integration surface $S$, respectively.

In the formulization of edge-based FEM, the second term on the right side of (4) is neglected because the values of the tangential components of the magnetic field $\boldsymbol{H}$ should be regarded as identical on the interface between the adjacent two elements:

$\int_{S} v \boldsymbol{n} \cdot[(\nabla \times A) \times w] \mathrm{d} S=\int_{S}(H \times w) \cdot n \mathrm{~d} S$.

However, during the computation, these values slightly differ between each other. Therefore, one could use these differences as an error estimator in the adaptive meshing method.

Let us consider the elements $i$ and $j$ having the common interface $S$ as shown in Fig. 3. This interface consists of the edges $k, l$, and $m$, and their vector interpolation functions $\boldsymbol{w}_{k}$, $\boldsymbol{w}_{l}$, and $\boldsymbol{w}_{m}$, respectively. Thus, the expression of the right term in (5) with respect to the edge $k$ becomes:

$d_{e, k}=\int_{S}\left(\boldsymbol{H}_{e} \times \boldsymbol{w}_{k}\right) \cdot \boldsymbol{n}_{e} \mathrm{~d} S \quad(e=i, j)$.

Similarly, two equations per edge could be written, while the following equations with respect to all three edges have to hold:

$D_{f}=d_{i, f}+d_{j, f}=0 \quad(f=k, l, m)$

In the ordinary edge-based FEA, as a result of neglecting of the second right term in (4), the values for $D_{k}, D_{l}$, and $D_{m}$ should be zero. However, in our case during the computation they are not zero and thus we utilize the calculated values for $D_{k}, D_{l}$, and $D_{m}$ as an error estimator $E_{i j}$ as following:

$E_{i \bar{y}}=\max \left(\left|D_{k}\right|,\left|D_{l}\right|,\left|D_{m}\right|\right)>\varepsilon$

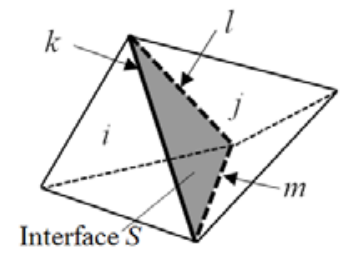

Fig. 3. The adjacent elements $i$ and $j$ with the common interface $S$. The triangular interface $S$ consists of the edges $j, k$, and $l$. 
where $\varepsilon$ is the prescribed threshold. If $E_{i j}$ exceeds the threshold $\varepsilon$, the elements $i$ and $j$ are adaptively subdivided into smaller elements.

\section{Proposed meshing refinement method utilizing non- conforming finite element technique}

Let us suppose that an element has a large error as shown in Fig. 4(a), and it has to be subdivided into eight smaller finite elements. Obviously, the elements surrounding the element with large error remain unchanged, resulting in generation of non-conforming element surfaces between the elements. If the initial shape of the parent element before subdivision was well-shaped, then also the shapes of the newly generated eight finite elements could remain well-shaped.

Three kinds of non-conforming techniques have already been proposed: (1) the discontinuous Galerkin method [8], (2) the mortar FEM [9], and (3) the mesh interpolating method [10], and in this paper we use the third one.

In the non-conforming finite element technique, the vector potential on master edges is obtained from slave edges as shown in Fig. 4(b). The original FEA equation is:

\section{$\boldsymbol{K} \boldsymbol{a}=\boldsymbol{b}$}

where $\boldsymbol{K}, \boldsymbol{a}$, and $\boldsymbol{b}$ are the stiffness matrix, the vector potential, and the source vector, respectively. The vector potential on slave edges $\boldsymbol{a}$ has the following relation with that on the master edge $\tilde{\boldsymbol{a}}$ :

$$
a=C \tilde{a}
$$

where $\boldsymbol{C}$ is the constitutional matrix derived from the relation between the master and slave edges [10]. The following system of equations needs to be solved:

\section{$C^{\prime} K C \tilde{a}=C^{\prime} b$}

where t stands for transpose, and $\boldsymbol{C}^{\mathrm{t}}$ is introduced in order to generate a symmetrical stiffness matrix.

In the proposed adaptive meshing method, a several iterative steps are taken for mesh refinement until an error $E_{i j}$ becomes below a defined tolerance value $\varepsilon$. Therefore, after the $i$ th refinement iteration step the following system is derived:

$$
C_{1}^{1} \cdots C_{i}^{1} K_{i} C_{i}^{1} \cdots C_{1}^{1} \tilde{a}_{i}=C_{1}^{1} \cdots C_{i}^{1} b_{i}
$$

where $\boldsymbol{C}_{i}$ is the constitution vector between the master and slave edges at the ith adaptive subdivision step. The system of linear equations (12) at the $i$ th adaptive subdivision step needs to be solved, and the constitutional vectors $C_{j}(j=1, \cdots, i-1)$ of

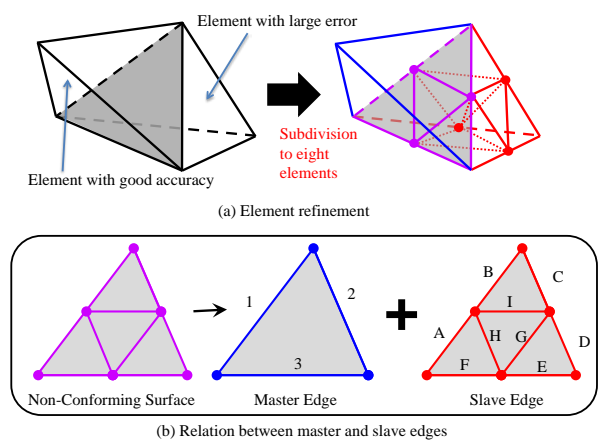

Fig. 4. (a) Subdivision of one element with large error to eight smaller elements, and (b) relation between master and slave edges on nonconforming surface. the previous steps could be reused to reduce a labor task. As for the stiffness matrix $\boldsymbol{K}_{i}$ and the source vector $\boldsymbol{b}_{i}$, it is just necessary to add their elements with respect to the newly subdivided elements to $\boldsymbol{K}_{i-1}$ and $\boldsymbol{b}_{i-1}$ at the previous step.

The main features of proposed mesh refinement method are:

- Easier subdivision into smaller elements. Just one tetrahedral element is simply subdivided into eight tetrahedral elements with high shape quality.

- The matrix elements of newly generated elements on every adaptive step are only added to the constitutional vector, the stiffness matrix, and the source vector, while the constitutional vectors on the previous steps are reused. After estimation of the computation error, it is not necessary to take into account the surfaces of objects during the mesh refinement procedure.

\section{APPLICATION}

To confirm the validity of the proposed adaptive method, we performed edge-based FEA on a simple model of a single permanent magnet (1T) as shown in Fig. 5, using a very rough tetrahedral initial mesh with 1325 elements. The mesh refinement behavior and accuracy transition of the proposed method were compared with those of the ZZ method. To keep approximately the same level of increasing rate of elements during mesh refinement for both, the proposed and the $\mathrm{ZZ}$ methods, only $10 \%$ of elements were allowed for subdivision per iteration.

\section{A. Mesh refinement}

In Figs. 6 and 7 the distribution of newly subdivided elements during iterative adaptive meshing process by the proposed and by the $\mathrm{ZZ}$ methods, respectively, are presented.

As shown in Fig. 6, the proposed method results into a finer mesh as expected mainly in the area around the permanent magnet. At earlier steps (Figs. 6(a) and (b)), the elements all over analysis area are subdivided. At the following steps (Figs. 6(c) and (d)), only finite elements belonging to the permanent magnet region and the air region surrounding the permanent magnet are evaluated. At the final step, only the elements near the outside surfaces of permanent magnet and the air are subdivided. Throughout the refinement process with the proposed method, only elements belonging in the area with large magnetic field gradient are chosen for subdivision.

In comparison, in case of the $\mathrm{ZZ}$ method the air region elements are subdivided in each step throughout the entire refinement process, as can be seen in Fig. 7. At the first step, the elements all over the analysis region are subdivided. However, at the second step, some elements on the top and

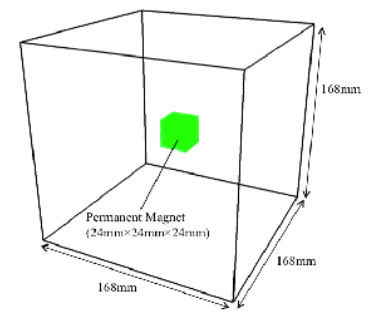

Fig. 5. Simple model of a single permanent magnet (1T). 


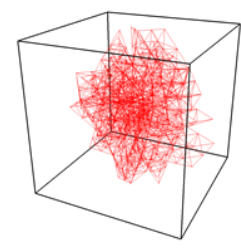

(a) 1 st

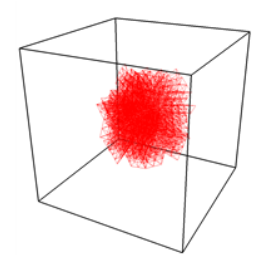

(b) 2nd

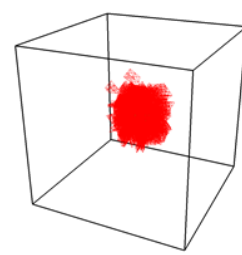

(c) 3rd

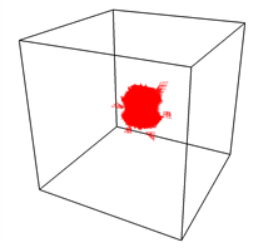

(d) 4 th

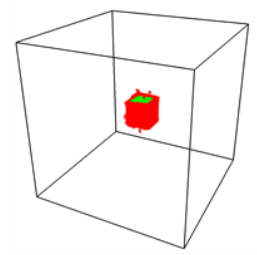

(e) 5 th

$1325 \rightarrow 2767$ elements $\quad 2767 \rightarrow 8276$ elements $\quad 8276 \rightarrow 28821$ elements

$28821 \rightarrow 71864$ elements $71864 \rightarrow 146050$ elements

Fig. 6. Element subdivision map as the adaptive step proceeds in the proposed adaptive meshing method. The red-highlighted elements mean subdivided elements at each refinement step.

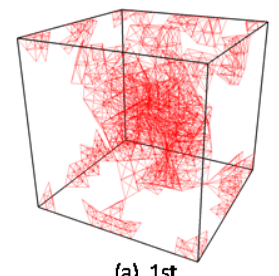

$1325 \rightarrow 2492$ elements

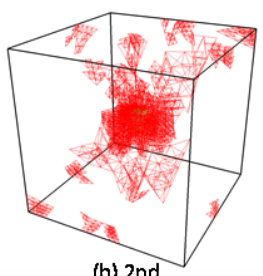

$2492 \rightarrow 6155$ elements

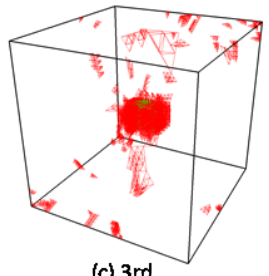

6155 - 13834 elements

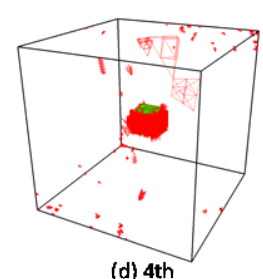

$13834 \rightarrow 36892$ elements

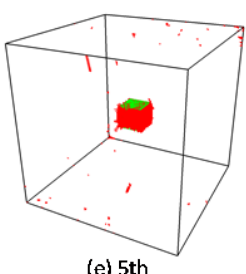

(e) 5 th

37892 -> 116083 elements

Fig. 7. Element subdivision map as the adaptive step proceeds in $\mathrm{ZZ}$ method. The red-highlighted elements are subdivided elements at each refinement step.

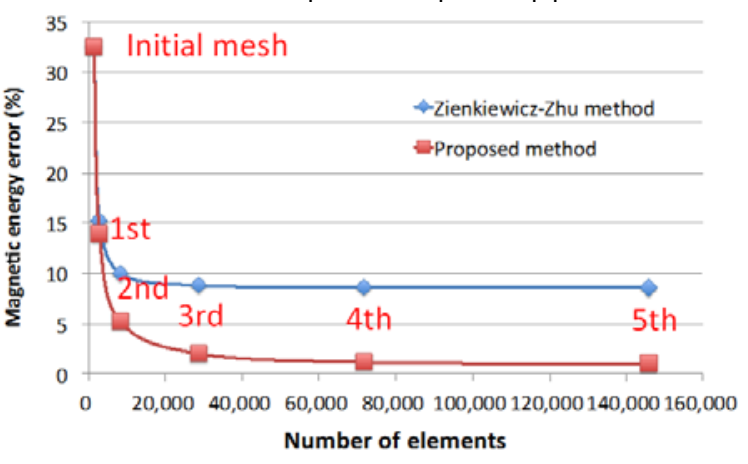

Fig. 8. Transition of magnetic field energy with increase of the number of elements in cases of the proposed adaptive meshing and $\mathrm{ZZ}$ method.

bottom surfaces and corners of analysis region are also evaluated as elements with large error, and consequently considered as a subject for subdivision. Even more, at the 3rd, 4th, and 5th steps, still elements far from the permanent magnet are also unnecessary subdivided (see Fig. 7(e)).

\section{B. Simulation accuracy}

Next, the accuracy of the obtained results is compared between the proposed and ZZ error estimation methods. Fig. 8 shows the transition of magnetic field energy error as the adaptive steps proceed in both, the proposed and ZZ methods.

The computation error using rough initial mesh was 32.6\%. Using the proposed method, the error at 5th step finally decreases to $1.0 \%$ with 146,050 elements. The computation error of the ZZ method decreases to $8.6 \%$ at the 4th step, and remained unchanged to the 5th step, which leads to the main conclusion that the proposed method provides high-quality mesh with less number of elements and better accuracy, than that of the traditional $\mathrm{ZZ}$ method.

\section{CONCLUSION}

In this paper, we have applied an error estimator based on the magnetic field conservation and a mesh improvement methodology using the non-conforming edge-based FEM to develop a new adaptive meshing method. The previously proposed error estimator based on the magnetic field conservation, in this paper, was extended and implemented to the adaptive meshing FEA. The proposed adaptive meshing generates better quality elements surrounding an object. As a result, the magnetic field error decreases from $32.6 \%$ to $1.0 \%$, showing superiority to the traditional $\mathrm{ZZ}$ error estimation method.

In the future, authors intend to apply the proposed method to non-linear and magneto-dynamic problems utilizing hexahedral edge-based finite elements.

\section{REFERENCES}

[1] J.-F. Remacle, P. DUlar, A. Genon, and W. Legros, "A posteriori error estimation and adaptive meshing using error in constitutive relation," IEEE Trans. Magn., vol. 32, no. 3, pp. 1369-1372, May 1996.

[2] S. Matsutomo, T. Miyamoto, K. Kaneda, S. Noguchi, and H. Yamashita, "An error evaluation scheme based on rotation of magnetic field in adaptive finite element analysis," IEEE Trans. Magn., vol. 42, no. 4, pp. 567-570, Apr. 2006.

[3] O. C. Zienkiewicz and J. Zhu, "The superconvergent patch recovery and a posteriori error estimators. Part 1: The recovery technique,” Int. J. Numer. Methods Eng., vol. 33, pp. 1331-1364, 1992.

[4] Z. Tang, Y. L. Menach, E. Creuse, S. Nicaise, F. Piriou, and N. Nemitz, "Residual and equilibrated error estimators for magnetostatic problems solved by finite element method,” IEEE Trans. Magn., vol. 49, no. 12, pp. 5715-5723, Dec 2013.

[5] R. Beck, R. Hiptmair, R. H. Hoppe, and B. Wholmuth, "Residual based a posteriori error estimators for eddy current computation," ESAIM: Math. Mode. Numer. Anal., vol. 34, no. 1, pp. 159-182, 2000.

[6] S. Nicaise and E. Creuse, "A posteriori error estimation for the heterogeneous Maxwell equations on isotropic and anisotropic meshes," Calcolo, vol. 40, no. 4, p. 249-271, 2003.

[7] C. Golovanov, J.-L. Coulomb, Y. Marechal, and G. Meunier, "3D mesh connection techniques applied to movement simulation," IEEE Trans. Magn., vol. 34, no. 5, pp. 3359-3362, Sep. 1998.

[8] S. Außerhofer, O. Bíró, and K. Preis, "Discontinuous Galerkin finite elements in time domain eddy-current problems," IEEE Trans. Magn., vol. 45, no. 3, pp. 1300-1303, Mar. 2009.

[9] T. Matsuo, Y. Ohtuski, and M. Shimasaki, "Efficient linear solvers for mortar finite-element method," IEEE Trans. Magn., vol. 43, no. 4, pp. 1469-1473, Apr. 2007.

[10] S. Bohmer, C. Kruttgen, B. Riener, and K Hameyer, "Eddy currents and non-conforming sliding interfaces for motion in 3-D finite element analysis of electrical machines," IEEE Trans. Magn., vol. 51, no. 3, Mar. 2015, Art. ID. 8103104. 